\title{
Gauge Invariance of Nonlinear Klein-Gordon
}

\author{
T. B. Prayitno \\ Theoretical Physics Group, Physics Department, Universitas Negeri Jakarta, Jl. Pemuda \\ Rawamangun No. 10 \\ Email : trunk_002@yahoo.com
}

\begin{abstract}
We have discussed the gauge invariance of nonlinear Klein-Gordon equation which describes the interaction of electromagnetic initially proposed by Hermann Weyl. The construction of nonlinear Klein-Gordon itself is formulated by two classical conservation laws, HamiltonJacobi of relativistic motion and continuity equations. Generally, this equation takes the similar concept to the derivation of the nonlinear master Schrödinger ignoring two fundamental concepts in ordinary quantum mechanics, Einstein and de Broglie's postulates. In this paper, the proposing for general form of nonlinear Klein-Gordon including the external potential and its gauge invariance have been established. However, we have to ignore the external potential in order to satisfy gauge invariance. In addition, we also prove the derivation of nonlinear Dirac equation for including half-integer spin concept can not hold by similar derivation.
\end{abstract}

\section{Keywords: nonlinear Klein-Gordon, gauge invariance.}

\section{Introduction}

The definition and properties of the nonlinear master Schrödinger equation have been discussed in the recent letters [1-6] which ignored some aspects, such as relativity, spin of particle, and the interaction term. In this paper, we have discussed that the construction for the relativistic motion of particle can be built by the same analogy in derivation of nonlinear master Schrödinger with the additional terms, called quantum potentials. Therefore, we define the nonlinear Klein-Gordon which is formulated by the similar assumption in the construction of nonlinear master Schrödinger equation without considering two fundamental postulates in ordinary quantum mechanics, Einstein and de Broglie's postulates, quantization of energy and momentum. Actually, for constructing the equation, we still start to ignore the concept of particle spin, such as in ordinary Klein-Gordon equation.

In the recent letters [1-6], which is summarized some of the present works, the nonlinear master Schrödinger was built from two classical balance equations, the Hamilton-Jacobi and continuity equations, which assumed that those two equations were still hold in the microscopic level. In ordinary KleinGordon equation, it is built by combination between two important postulates in ordinary quantum mechanics and the relativistic energy equation. In this paper, we did not use those two expressions to build the nonlinear Klein-Gordon, but we will demonstrate that the nonlinear KleinGordon can be derived by Hamilton-Jacobi of relativistic motion and continuity equation.

The ordinary Klein-Gordon itself represents the relativistic free particle motion equation. In this case, we also can show that we can always include the interaction term for the generalization of nonlinear Klein-Gordon equation. This interaction term is included from the Hamilton-Jacobi equation, such as in the classical physics. However, we also get the same problem in the ordinary KleinGordon since our equation contains the negative energy.

The rest of the paper is arranged as follow: in section II we discuss the derivation of general nonlinear Klein- 
Gordon by extending the Hamilton-Jacobi inserting the external potential. In section III we construct the form of gauge invariance of nonlinear Klein-Gordon based on the local gauge theory ${ }^{1}$ from Hermann Weyl's idea [7] describing the interaction of electromagnetic fields. This procedure is same as the construction of ordinary Klein-Gordon equation applying phase transformation in the wave function. Unfortunately, the external potential term must be ignored for preserving gauge invariance. In addition, we have to modify the Hamilton-Jacobi including the electromagnetic fields.

For the final discussion, we finally prove the failure for constructing nonlinear Dirac equation by similar way in order to include half-integer spin concept. The inserting of that spin concept is important for describing the motion of electron and its interaction. For the closing term, we present our conclusions based on the results.

\section{Derivation Of Nonlinear Klein- Gordon And Its General}

In the recent letter [1], the derivation for constructing the nonlinear master Schrödinger did not use the two famous postulates principle in quantum mechanics, but it used the HamiltonJacobi and continuity equation which have been postulated before. For the similar way, we also use those two basic equations for constructing the nonlinear Klein-Gordon. For the simplicity, we start from Hamilton-Jacobi of relativistic motion and continuity equation which have been defined before ${ }^{2}$

$$
\begin{aligned}
& \frac{1}{c^{2}}\left(\frac{\partial \varphi}{\partial t}\right)^{2}-(\nabla \varphi)^{2}-m^{2} c^{2}=0, \\
& \nabla \cdot\left(a^{2} \nabla \varphi\right)=\frac{1}{c^{2}} \frac{\partial}{\partial t}\left(a^{2} \frac{\partial \varphi}{\partial t}\right),
\end{aligned}
$$

\footnotetext{
${ }^{1}$ This theory was initially proposed by Hermann Weyl as the symmetry of Maxwell's equations in classical electrodynamics

${ }^{2} \varphi$ means the classical action in classical physics.
}

where $c$ and $m$ mean light velocity and rest mass of particle. After making certain algebra and combining those two equations, one gets the final expression, named nonlinear Klein-Gordon

$$
\begin{aligned}
\frac{1}{c^{2}} & \frac{\partial^{2} \psi}{\partial t^{2}}-\nabla^{2} \psi+\frac{m^{2} c^{2}}{\hbar^{2}} \psi \\
& -\frac{1}{c^{2} a} \frac{\partial^{2} a}{\partial t^{2}} \psi+\frac{\nabla^{2} a}{a} \psi=0,
\end{aligned}
$$

where $\psi$ is the general solution written in expression

$$
\psi(\vec{r}, t)=a(\vec{r}, t) e^{\frac{i}{\hbar} \varphi(\vec{r}, t)} .
$$

The expression (3) describes the relativistic motion of free particle and the nonlinear terms are called quantum potentials.

The next purpose is to build the general nonlinear Klein-Gordon which includes the interaction term. The possibility for that construction is to generalize the Hamilton-Jacobi of the relativistic motion in expression (1) as [8].

$$
\frac{1}{c^{2}}\left(\frac{\partial \varphi}{\partial t}-V\right)^{2}-(\nabla \varphi)^{2}-m^{2} c^{2}=0
$$

where $\mathrm{V}$ is identified as an external potential term. After that, we have to combine together with the continuity equation defined in Eq. (2).

In such condition, after using a little algebra, we will get the general form of nonlinear Klein-Gordon equation as

$$
\begin{aligned}
& \frac{1}{c^{2}} \frac{\partial^{2} \psi}{\partial t^{2}}-\nabla^{2} \psi+\frac{m^{2} c^{2}}{\hbar^{2}} \psi-\frac{1}{c^{2} a} \frac{\partial^{2} a}{\partial t^{2}} \psi \\
& +\frac{\nabla^{2} a}{a} \psi+\left(\frac{2}{\hbar^{2} c^{2}} \frac{\partial \varphi}{\partial t}-\frac{1}{c^{2} \hbar^{2}}\right) V \psi=0,
\end{aligned}
$$

where $\mathrm{V}$ describes the interaction term. However, as stated before, we remind that the general solution for that equation above still has form in Eq. (4). 


\section{Gauge Invariance Form}

In the several textbooks [9-10], the gauge invariance of ordinary KleinGordon has been established. The gauge invariance itself is based on the local gauge initially proposed by Hermann Weyl taken from the analogy of connection in general relativity. In this section we focus to construct the gauge invariance of nonlinear Klein-Gordon equation. By seeing the expression (6), we can guess that the way for deriving the gauge invariance of ordinary KleinGordon should be applied of nonlinear Klein-Gordon either.

Since the wave solution of nonlinear Klein-Gordon has the general amplitude depends on space and time, it is clear that we have to transform the corresponding amplitude by local gauge transformation

$$
a(\vec{r}, t) \rightarrow a^{\prime}(\vec{r}, t)=e^{i \theta} a(\vec{r}, t),
$$

with the $\theta$ parameter is the function of space and time. In the other hand, by similar analogy, we generalize the differential operators as follow [11].

$$
\begin{aligned}
& \nabla \rightarrow \vec{D}=\nabla-i q \vec{A}, \\
& \frac{\partial}{\partial t} \rightarrow D_{t}=\frac{\partial}{\partial t}+i q \phi .
\end{aligned}
$$

In this case, we initially state that $\phi$ and

$\vec{A}$ are unknown potentials ${ }^{3}$.

If we make the condition for the amplitude

$$
\begin{aligned}
& D_{t}^{\prime} a^{\prime}=e^{i \theta} D_{t} a, \\
& \vec{D}^{\prime} a^{\prime}=e^{i \theta} \vec{D} a,
\end{aligned}
$$

we can easily show that the generalization of nonlinear Klein-Gordon will be invariant under simultaneous transformation

$$
\begin{aligned}
& a(\vec{r}, t) \rightarrow a^{\prime}(\vec{r}, t)=e^{i \theta} a(\vec{r}, t), \\
& \vec{A} \rightarrow \vec{A}^{\prime}=\vec{A}+\frac{1}{q} \nabla \theta, \\
& \phi \rightarrow \phi^{\prime}=\phi-\frac{1}{q} \frac{\partial \theta}{\partial t} .
\end{aligned}
$$

\footnotetext{
${ }^{3}$ Finally, we recognize that those two potentials are scalar and vector potentials in classical electrodynamics
}

if only if the interaction potential should be zero, $V=0^{4}$. In other words, in the presence of electromagnetic field, the nonlinear Klein-Gordon should be

$$
\begin{aligned}
\frac{1}{c^{2}} D_{t}^{2} \psi-\vec{D}^{2} \psi+\frac{m^{2} c^{2}}{\hbar^{2}} \psi & \\
-\frac{1}{c^{2} a}\left(D_{t}^{2} a\right) \psi+\frac{\vec{D}^{2} a}{a} \psi & =0 .
\end{aligned}
$$

\section{Failure of Construction Nonlinear Dirac Equation}

In quantum field theory, Dirac proposed the equation of relativistic particle motion in order to include the half-integer spin concept by factorizing ordinary Klein-Gordon and introducing Gamma matrices containing Pauli spin matrices. According to him, Dirac equation can be written as

$$
\frac{i \hbar}{c} \gamma^{0} \frac{\partial \psi}{\partial t}+i \hbar \vec{\gamma} \cdot \nabla \psi-m c \psi=0 \text {, }
$$

with gamma matrices are defined

$$
\gamma^{0}=\left(\begin{array}{cc}
1 & 0 \\
0 & -1
\end{array}\right), \vec{\gamma}=\left(\begin{array}{cc}
0 & \vec{\sigma} \\
-\vec{\sigma} & 0
\end{array}\right),
$$

and $\vec{\sigma}$ is a standard vector form of Pauli spin matrix (all components of Pauli spin matrices will be given in appendix).

It has been discussed that the construction of nonlinear Klein-Gordon is built by adding the quantum potential terms achieved by expanding the general wave function and concerning only in real component. The potentials are the terms containing Planck constant, $\hbar$, in real component. By this idea, we follow the instruction given above by writing the general solution in Eq. (4) into Eq. (16). After separating the real and imaginary components, we find

\footnotetext{
${ }^{4}$ It is clear since the phase term, $\varphi$, was not transformed under local gauge transformation
} 
- real component

$\frac{1}{c} \gamma^{0} \frac{\partial \varphi}{\partial t}+\vec{\gamma} \cdot \nabla \varphi+m c=0$,

- imaginary component

$\frac{1}{c} \gamma^{0} \frac{\partial a}{\partial t}+\vec{\gamma} \cdot \nabla a=0$.

Since the real component has no Planck constant term, it is clear that no quantum potential. We directly conclude that nonlinear Dirac equation can not be constructed by the method. Thus, we have proven that the inserting of halfinteger spin can not be built.

\section{Conclusion}

In this letter, the idea for establishing of the derivation of gauge invariance for general nonlinear Klein-Gordon equation has been established. This construction is based on the similar way to the construction of gauge invariance for nonlinear master Schrödinger which extends the definitions of differential operators. The construction itself has ignored two fundamental postulates in ordinary quantum mechanics, Einstein and de Broglie's postulates.

In addition, we have proven that the gauge invariance only hold if one removes the external potential term. This fact is a different case if one considers the general form of linear Klein-Gordon keeping the interaction term in quantum field theory. However, in this case we haven't made any prediction whether the solution for negative energy can be accepted or not.

To completely describe the characteristics of electron, one has to consider half-integer spin concept. The not interesting case is the failure of including that concept when extends the Dirac equation into nonlinear equation. We conclude this truth by considering Hamilton-Jacobi having no Planck constant term. Even though we get the failure, the idea is interesting because the main purpose of the construction is to build the general relativistic quantum mechanics initially proposed by Einstein and de Broglie in the early time.

\section{Acknowledgements}

It is a pleasure for us to acknowledge to all lecturers of physics department in Universitas Negeri Jakarta for the encouragements.

\section{Appendix}

In classical mechanics, the corresponding three-dimensional Hamilton-Jacobi of relativistic motion and continuity equations are generally defined $\frac{1}{c^{2}}\left(\frac{\partial S_{c l}}{\partial t}\right)^{2}-\left(\nabla S_{c l}\right)^{2}-m^{2} c^{2}=0,(20)$ $\frac{\partial \rho}{\partial t}+\nabla \cdot \vec{J}=0$,

where all of those symbols have the usual meaning. The reader can review for more detail in the following textbooks [12-14].

In discussion of nonlinear KleinGordon, those symbols are transformed into the appropriate wave quantities below

$$
\begin{aligned}
& S_{c l}=\varphi, \quad \vec{p}=\nabla \varphi=\gamma m \vec{v}, \\
& E=-\frac{\partial \varphi}{\partial t}=\gamma m c^{2}, \rho=a^{2}, \vec{J}=\rho \vec{v},
\end{aligned}
$$

where

$$
\gamma=\frac{1}{\sqrt{1-\frac{v^{2}}{c^{2}}}} .
$$

is the famous Lorentz contraction. In our convention, all the components of Pauli spin matrices are given by

$$
\begin{aligned}
& \sigma_{x}=\left(\begin{array}{cc}
0 & 1 \\
1 & 0
\end{array}\right) ; \sigma_{y}=\left(\begin{array}{cc}
0 & -i \\
i & 0
\end{array}\right) ; \\
& \sigma_{z}=\left(\begin{array}{cc}
1 & 0 \\
0 & -1
\end{array}\right)
\end{aligned}
$$




\section{References}

1. F. Guerra and M. Pusterla, Lett. Nuovo Cimento, 34, 1982, 351.

2. Ph. Gueret and J. P. Vigier, Lett. Nuovo Cimento, 38, 1983, 125.

3. L. Smolin, Phys. Lett. A 113, 1986, 408.

4. J. P. Vigier, Phys. Lett. A 135, 1989, 99.

5. T. B. Prayitno, Jurnal Fisika dan Aplikasinya (Spektra), Vol. IX No. 2 Desember 2010.

6. J. R. Croca, Towards a Nonlinear Quantum Physics, Singapore : World Scientific, 2003, pp. 65-82.

7. K. Moriyasu, An Elementary Primer for Gauge Theory, 2nd ed., Singapore: World Scientific, 1983, pp. 5-14.

8. L. de Broglie, An Introduction to the Study of Waves Mechanics, translated by L. T. Flint, Paris : Methuen \& Co. Ltd, First published in 1930, pp. 79-87.

9. G. Kane, Modern Elementary Particle Physics, $1^{\text {st }}$ ed., Michigan : Westview Press, 1993, pp. 35-40.

10.L. H. Ryder, Quantum Field Theory, $2^{\text {nd }}$ ed., Cambridge University Press, New York, 1996, pp. 81-97.

11.D. J. Griffiths, Introduction To Electrodynamics, 2nd ed., New Jersey: Prentice-Hall, 1999, pp. 416-421.

12.Walter Greiner, Classical Mechanics (System of Particles and Hamiltonian Dynamics), : Springer-Verlag, 2003, pp. 386-409.

13.W. Dittrich and M. Reuter, Classical and Quantum Dynamics, Berlin : Springer-Verlag, 1996, pp. 62-73.

14.H. Goldstein, Classical Mechanics, 3rd ed., Addison Wesley, New York 2000, pp. 430-439. 